\title{
Heart Surgery Access Bone: Spectrum of Pathology
}

\author{
Khaled Ebrahim Al-Ebrahim, FRCSC
}

Department of Cardiothoracic Surgery, King Abdulaziz University, Jeddah, Saudi Arabia

\section{ABSTRACT}

A variety of pathologies may affect the most important bone to cardiac surgeons. In this paper, 3 interesting cases of different pathology and aetiology are presented and discussed.

\section{INTRODUCTION}

Median sternotomy is considered the benchmark incision for cardiac surgery, enabling the surgeon to have a better view of the heart, great vessels, and lungs. It is considered the most common osteotomy performed worldwide.

Clinical summary: Case No. 1 - complete sternal cleft in an adult.

A 35-year-old female presented with complete sternal cleft, causing a cosmetic concern and constant fear of trauma. Physical examination showed visible aortic and cardiac pulsations. There was complete sternal cleft that measured 6 $\mathrm{cm}$ in width between the remnants of the sternal edges in inspiration and $4 \mathrm{~cm}$ in expiration. During Valsalva maneuver, the sternal cleft became wider, and the heart bulge was more prominent. Echocardiography showed no congenital defects. Computerized tomograms were done and confirmed the diagnosis (Figure 1).

Intraoperatively, the mediastinal tissues covering the pericardium carefully were dissected to expose the anterior and posterior aspect of the sternal body edges. Subcutaneous skin flaps were raised on both sides to expose the manubrial ends and the upper four costal cartilages and ribs. The under surface of the sternum and ribs also were mobilized.

The first trial of test approximation of the sternal edges resulted in signs of cardiac compression. Widely opening the left pleural cavity and second trial of gradual and slow approximation did not show any hemodynamic changes.

Titanium plates were used to close the defect. A star shaped titanium plate was used in the manubrium, and 2 rows were applied to the third and fourth costal cartilages. Each plate on either side was fixed with 3 predrilled screws (Figure 2). Postoperative course was smooth and the patient was discharged home well. The patient has been followed for more than 5 years with no complications (Figure 3).

Received February 20, 2020; accepted March 6, 2020

Correspondence: Khaled E. Al-Ebrabim, Department of Cardiothoracic Surgery, King Abdulaziz University Hospital, P.O. Box 80215, Jeddah 21589 Saudi Arabia; fax+026408347; +026401000 (e-mail:dr.k.ebrabim@gmail.com).
Case No. 2 - polyostotic fibrous dysplasia of the sternum

A 36-year-old female presented with a painless sternal mass $3.2 \times 4.5 \mathrm{~cm}$ non-tender, hard, and immobile, arising from the body of the sternum. She also complained of tenderness along the left fourth ribs. Computerized tomography (CT) scans and magnetic resonance imaging (MRI) of the chest showed expansile, osteolytic and hyper translucent
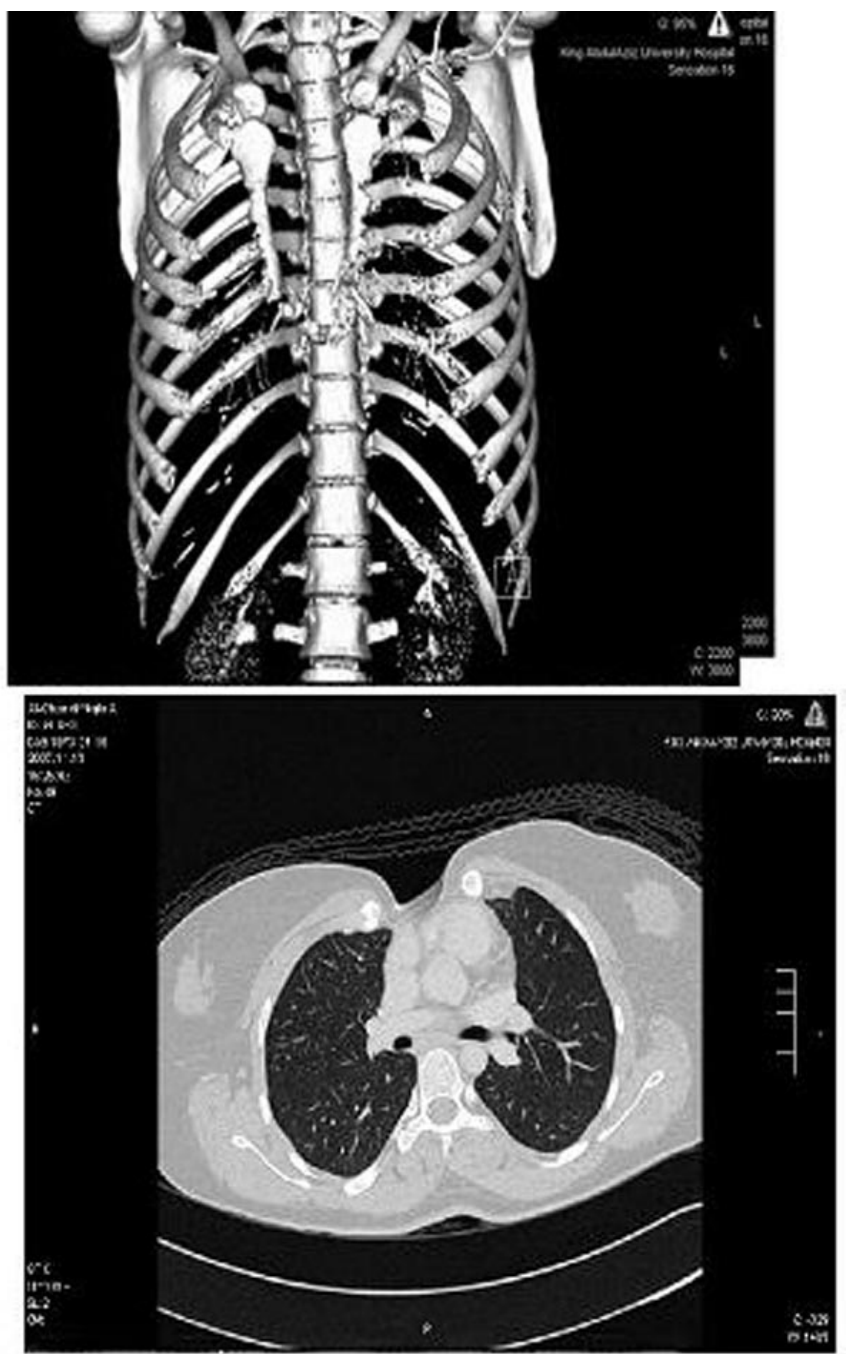

Figure 1. Top: 3D computerized tomography reconstruction showing the complete sternal cleft. Bottom: Cross section CT image at the sternal defect level. 


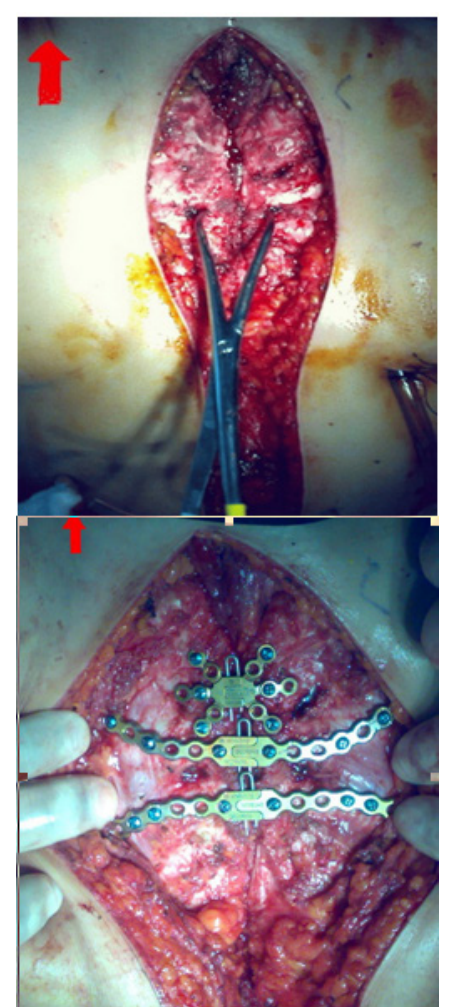

Figure 2. Intraoperative titanium plate closure of the defect.

multilocular septated lesions of the sternum, the fourth rib and the fourth thoracic vertebral body (Figures 4 and 5). Bone scan revealed intense heterogeneous isotope uptake in the same sites. Hormonal assay to rule out the McCune-Albright syndrome were within normal limits. Open wedge resection of the tumor in the sternal body revealed fibrous tissue proliferation arranged in storiform pattern. Trabeculae of woven bone lacking the osteoblastic rimming are arranged like Chinese letters or alphabet soup, typical of fibrous dysplasia. The sternal involvement conservatively was treated, and the patient was referred to neurosurgery for vertebral stabilization to avoid collapse fracture and spinal complications.

Case No. 3 - sternal lymphoma

A 67-year-old man was referred to us with a painful sternal mass one-month duration. On examination a firm, fixed, tender $6 \times 10 \mathrm{~cm}$ sternal mass was involving the upper half of the sternal body. Chest $\mathrm{x}$-ray and computed tomographic studies of the chest demonstrated a $5.2 \times 5.7 \times 6 \mathrm{~cm}$ mass originating in the left side of the sternum. No extension into the mediastinum and no mediastinal or hilar adenopathy were present. The lung fields were clear, and the large airways were without compromise. Computed tomographic scans of the head, abdomen, and pelvis, a radionuclide bone scan, showed 2 hypodense areas in the liver.

At operation, a longitudinal median incision over the mass, biopsy with frozen section demonstrated undifferentiated anaplastic tumor vs lymphoma, and the patient underwent surgical resection of the mass with a $4 \mathrm{~cm}$ safety margin. The lower end of the manubrium and the upper half of sternum were resected from the first to the fourth intercostal spaces,

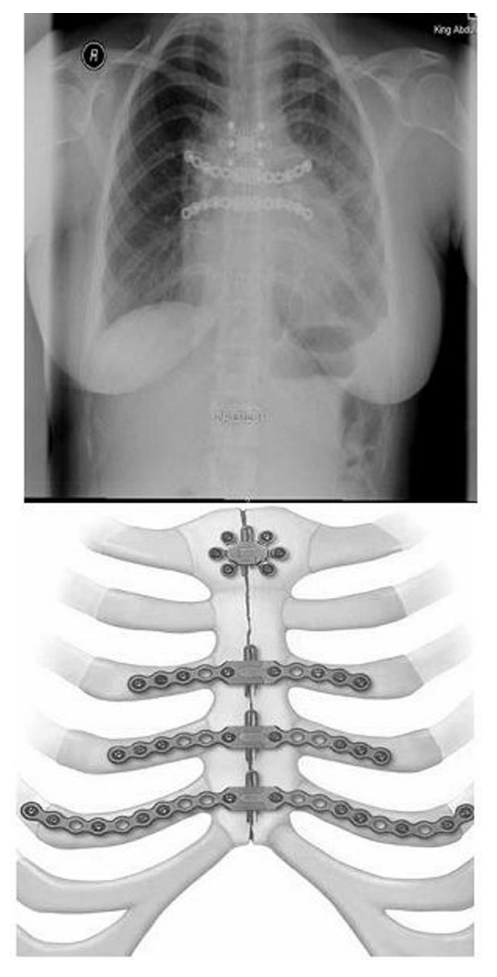

Figure 3. Postoperative chest $x$-ray showing the titanium plates.

including the second and third costal cartilages bilaterally. The tumor was excised with dissection of the mediastinum fat and mammary lymph node. Soft tissue reconstruction with pectoralis muscles and overlying skin was satisfied. Pathologic examination of the specimen demonstrated a soft pinktan lesion originating in the sternum, replacing the trabecular bone and thinning the cortical bone. The tumor did not extend to the mediastinum. Microscopic evaluation demonstrated sheets of malignant, highly pleomorphic lymphocytes within the sternal mass. Extensive necrosis and a high mitotic rate were present, confirming the diagnosis of a non-Hodgkin's immunoblastic B-cell lymphoma originating in the sternum. The patient received 6 cycles of cyclophosphamide, doxorubicin, vincristine, and prednisone and is well with no evidence of active disease 2 years after the operation.

\section{DISCUSSION}

Case No. 1 - Successful primary surgical repair of complete sternal cleft in an adult

Congenital sternal fissure or cleft is a very rare anomaly, accounting for less than 1 in 100,000 live births. It results from failure of ventral fusion of the sternum, which occurs in about the 8 th week of gestation. The complete type occurs in only about $20 \%$ where bone formation is absent between the hyoid and pubis [Ravitch 1983]. Sternal clefts, especially the complete ones, must be closed for protection of the heart and great vessels as those patients feel insecure in addition to the cosmetic appearance. 


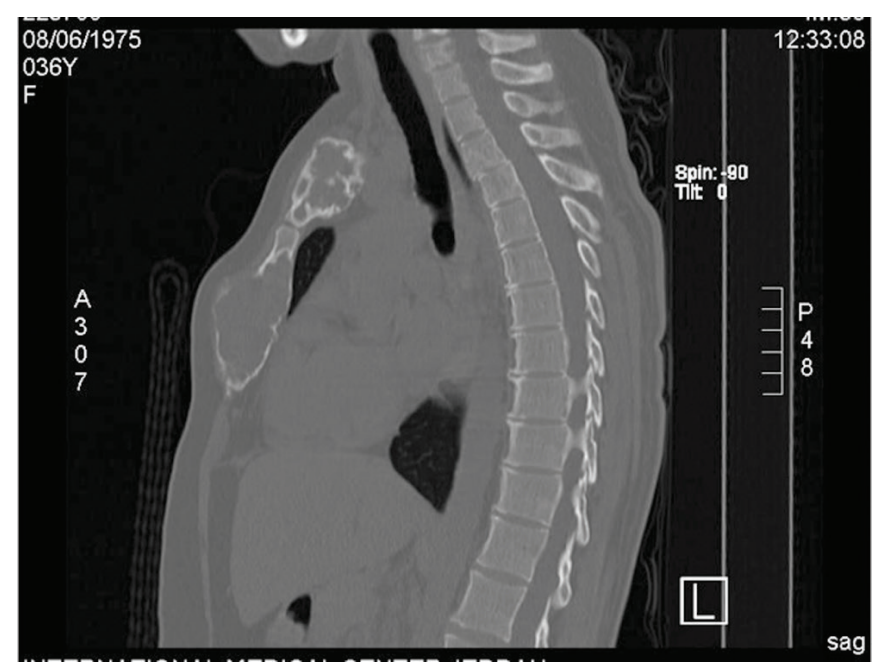

Figure 4. MRI sagittal view showing fibrous dysplasia of the manubrium and body of the sternum.

Few cases have been reported in the literature and most of them were partial clefts repaired in the neonatal period, which is the ideal time when the chest wall and thoracic cavity still are elastic and flexible. Most of the difficulty of closing these clefts in adults is attributed the rigidity of the chest wall, lack of the flexibility, and the need for sternal substitute to cover the missing bone. Most of the reported cases in the literature of complete cleft repair were done using either synthetic Marlex, Teflon or Prolene Mesh, methcrylate sandwich or autogenous tissues (iliac crest or rib grafts) covered by pectoralis major flaps [Voss 2008; Suri 1996]. The main draw backs of such complex sternal reconstruction is the huge amount of foreign body material with its liability to untoward reaction, infection, extra weight on the sternum, and the difficulty of going back to the heart or other mediastinal structures in the future for any surgical procedure.

Santini and colleagues reported a successful primary repair of complete sternal defect associated with congenital heart disease in a 52-year-old patient [Santini 1995]. Direct closure of sternal clefts associated with pectus excavatum is more feasible as the excision of the costal cartilages on both sides would facilitate approximation of the 2 sternal edges [Sarper 2002].

The sternal fixation system using plates is ideal for these patients; there is not enough sternal bone to use stainless steel wires. This system has been used in patients with mediastinitis and complicated sternal dehiscence instead of the conventional closure and have proven to be effective. The other advantage of this system is its locking and unlocking feature, which allows easy opening in re-exploration. The width of the cleft is the most important factor, determining the possibility of direct closure. In our case it was about $6 \mathrm{~cm}$ in inspiration, which is considered a fairly wide space, and we think that the gradual closure plus opening the left pleural cavity helped to avoid cardiac compression. The availability of sternal substitute reconstructive procedure must be there at all times, in case of intolerance to direct closure. In conclusion, simple sternal closure should be attempted in those patients before

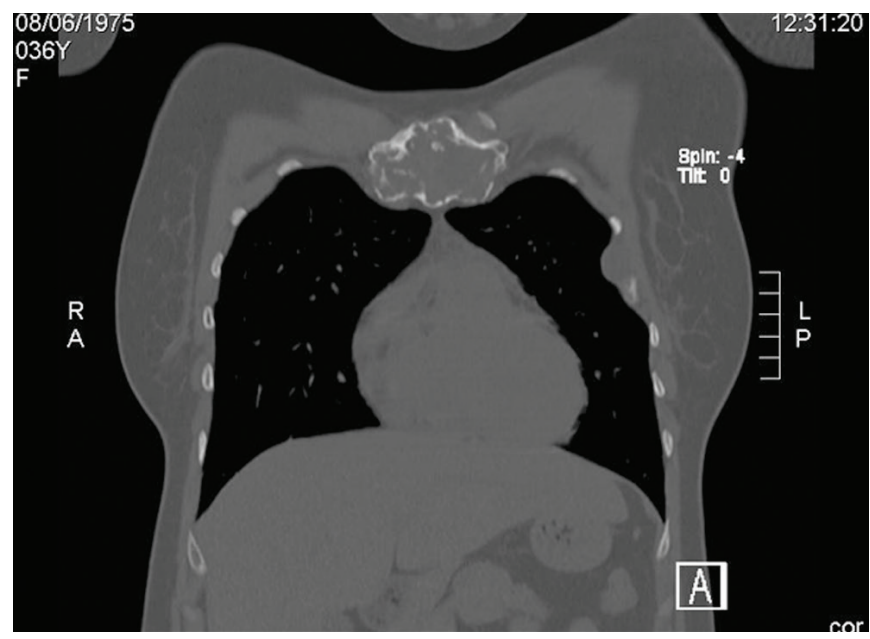

Figure 5. MRI coronal view showing the multi septated hyper translucent sternum.

embarking on a complex reconstruction with all its drawbacks.

Case No. 2 - sternal polyostosis (Chinese letter or alphabet soup tumor of the sternum)

Fibrous dysplasia is a congenital, non-inherited connective tissue disorder in which medullary bone is replaced with fibroosseous connective tissue [Siegal 2013]. The disorder can present in either a single skeletal site or multiple sites, respectively termed monostotic or polyostotic. The McCune-Albright syndrome is a severe form of fibrous dysplasia, which is associated with café-au-lait-spots and endocrine dysfunction. The primary etiology has been linked to a mutation in the alpha subunit of the guanine-nucleotide binding protein (Gs- $\alpha$ ), leading to continuous activation of adenylate cyclase and increased cyclic adenosine monophosphate (cyclic AMP) formation, leading to abnormal bone development [Leet 2004]. Long bones, lumbar spine, and craniofacial bones are the most common sites for fibrous dysplasia. The sternum is the rarest bone to be affected [Leet 2004]. Extensive chest wall involvement can lead to restrictive lung disease and cor pulmonale requiring release by chest wall reconstruction [Tang 2011; Narayan 2009]. Early cases like this require following up with periodical CT, MRI, or bone scan to monitor progression in size or eruption of new sites. Surgery is indicated in symptomatic patients, compression of vital organs, or fear of fracture.

Case No. 3 - sternal lymphoma

The management of sternal tumors may be complex and typically is directed by the histologic nature of the tumor, its location, and the extent of involvement of the sternum and adjoining structures. The majority of sternal tumors are malignant. Primary solid and small cell tumors, as well as metastatic lesions, have been reported [Li 2018].

Both Hodgkin's disease and non-Hodgkin's lymphoma involving the sternum and chest wall have been described. Frequently, the malignancy originates within lymph nodes of the mediastinum or internal thoracic chain and invades the chest wall through direct extension. Mediastinal adenopathy occurs frequently in Hodgkin's disease, but is unusual in nonHodgkin's lymphoma. Isolated chest wall masses distinctly 
are uncommon and usually are a manifestation of large cell non-Hodgkin's lymphoma. These tumors are extremely rare and most of the reported literature is case reports [Singh 2015; Ishizawa 1995].

Resection of sternal tumors with adequate surgical margins of $4 \mathrm{~cm}$ may result in substantial bony and soft tissue defects. Loss of the upper sternal body and the costal cartilages, as in this case, produces a defect that is not physiologically significant. More extensive resections, including the entire sternal body or manubrium with the adjacent ribs and clavicles, produce more severe deficits. Reconstruction therefore is necessary to avoid prolonged ventilatory support. Reconstruction after sternal resection can be performed with a variety of materials. Skin defects frequently are small and most often covered primarily by advancing skin flaps. Larger defects may require split-thickness skin grafting or closure as part of a myocutaneous tissue flap. Underlying soft tissue defects may be closed by advancing pectoralis muscle flaps, by transposition of a free tissue graft, or by an omental flap. Prosthetic materials, including polytetrafluoroethylene, methyl methacrylate, polypropylene, polyethylene, and steel mesh have all successfully been used in reconstructing sternal defects.

\section{CONCLUSION}

Awareness of different pathologies and lesions of the sternum is important to all cardiothoracic surgeons. Multiple procedures are available to secure solidity of this bone, which provides the most important protection to the heart and great vessels.

\section{ACKNOWLEDGMENTS}

This project was funded by the Deanship of Scientific Research (DSR), King Abdulaziz University, Jeddah, Saudi Arabia, under grant No. (1441-50-140) . The authors therefore, gratefully acknowledge the DSR technical and financial support.

\section{REFERENCES}

Ishizawa M, Okabe H, Matsumoto K, Hukuda S, Hodihara K, Ota S. 1995. Anaplastic large cell Ki-lymphoma with bone involvement: report of two cases. Virchows Arch 427:105-10.

Leet AI, Magur E, Lee JS, Wientroub S, Robey PG, Collins MT. 2004. Fibrous dysplasia of the spine: prevalence of lesions and association with scoliosis. J Bone Joint Surg Am 86-A(3): 531-537.

Li Y, Qin Y, Zheng L, Liu H. 2018. "Extranodal presentation of Hodgkin's lymphoma of the sternum: A case report and review of the literature". Oncology Letters 15, no. 2: 2079-2084.

Narayan RL, Maldjian PD. 2009. Restrictive lung disease and cor pulmonale secondary to polyostotic fibrous dysplasia. Int J Cardiol 131(2): e48-50.

Ravitch MM. 1983. Disorder of the sternum and the thoracic wall. In: Sabiston DC Jr, Spencer FC, eds. Gibbon's surgery of the chest, 4th ed. Philadelphia: Saunders, 318-60.

Santini F, Faggian G, Pessotto R, Mazzucco A. 1995. Successful repair of complete sternal cleft associated with congenital heart disease: report of one case. J Cardiovasc Surg 36:75-77.

Sarper A, Oz N, Arslan G, Demircan A. 2002. Complete Congenital Sternal Cleft Associated with Pectus Excavatum. Tex Heart Inst J. 293:206-209.

Siegal GP, Bianco P, Dal Cin P. 2013. Fibrous dysplasia. In: WHO Classification of Tumours of Soft Tissue and Bone, 4th Ed. Fletcher CDM, Bridge JA, Hogendoorn PCW, Mertens F. (Eds), International Agency for Research on Cancer, Lyon, France, 352.

Singh S, Jenaw RK, Jindal A, Bhandari C. 2015. Hodgkin's lymphoma presenting as lytic sternal swelling. Lung India. 32(4):410-412.

Suri RK, Sharma RK, Jha NK, Sharma BK. 1996. Complete congenital sternal cleft in an adult: repair by autogenous tissue. Ann Thoracic Surg 62:573-5.

Tang J, Wang JJ, Zhai W, Zhang SC. 2011. Chest wall reconstruction in a patient with sternal fibrous dysplasia. Thorac Cardiovasc Surg 59(1): 58-60.

Voss B, Bauenrschmitt R, Will A, Krane M, Kross R, Brockmann G, Libera P, Lange R. 2008. Sternal reconstruction with titanium plates in complicated sternal dehiscence. Eur J Cardiothrac Surg. 34: 139-145. 